\title{
Familial Apical Hypertrophic Cardiomyopathy in a Young Adult: A Rare Occasion for Making Precise Diagnostic in a Low Income Country
}

\author{
Sylvie Ndongo Amougou ${ }^{1,2}$, Helles Murielle Lema ${ }^{1}$, Mazou Ngou Temgoua ${ }^{1, ~ *}$, \\ Ngam Mary Engonwei ${ }^{1}$, Samuel Kingue ${ }^{1,3}$ \\ ${ }^{1}$ Department of Internal Medicine, Faculty of Medicine and Biomedical Sciences, University of Yaoundé, Yaoundé, Cameroon \\ ${ }^{2}$ Department of Internal Medicine, Yaoundé University Teaching Hospital, Yaoundé, Cameroon \\ ${ }^{3}$ Department of Internal Medicine, General Hospital of Yaoundé, Yaoundé, Cameroon
}

\section{Email address:}

ndongoamougou@yahoo.fr (S. N. Amougou), murielle.lema@gmail.com (H. M. Lema), neurotemgoua@yahoo.fr (M. N. Temgoua), ngammaryanne@yahoo.com (N. M. Engonwei), samuel.kingue@hotmail.fr (S. Kingue)

*Corresponding author

\section{To cite this article:}

Sylvie Ndongo Amougou, Helles Murielle Lema, Mazou Ngou Temgoua, Ngam Mary Engonwei, Samuel Kingue. Familial Apical Hypertrophic Cardiomyopathy in a Young Adult: A Rare Occasion for Making Precise Diagnostic in a Low Income Country. Cardiology and Cardiovascular Research. Vol. 3, No. 2, 2019, pp. 27-30. doi: 10.11648/j.ccr.20190302.12

Received: April 14, 2019; Accepted: May 23, 2019; Published: June 11, 2019

\begin{abstract}
Hypertrophic Cardiomyopathy is a genetic disorder with asymmetric left ventricular hypertrophy. In a low income country it is sometimes difficult to do global checkup and precise diagnosis in suspected patients. We present a case of apical hypertrophic cardiomyopathy with MYBPC 3 mutation in a young Cameroonian. A 36 years old man with no cardiovascular risk factors, presents with a progressive chest pain on exertion. The physical examination was normal. The resting electrocardiography showed inverted $\mathrm{T}$ waves in anterior, lateral and inferior leads. Treadmill electrocardiography and Coronarograpy were normal. A transthoracic cardiac ultrasound showed hypertrophy of $168 \mathrm{~mm}$ in the apical segment, the systolic function and the regional wall motion of the left ventricle were normal. We concluded of hypertrophic cardiomyopathy with abnormality in the myosin binding protein C (MYBPC3) found on genetic analysis. A screening cardiac ultrasound was realized in the patient's family and the son was found to have septal hypertrophy. Strict follow-ups were organized for the patient and his son. Familial Hypertrophic Cardiomyopathy is a rare disease in cardiology; the precise diagnosis requires complex exams which are sometimes unavailable in low income countries. This case was a special one with all necessary investigations giving the possibility to organize follow-ups for patient and related family member to prevent sudden death.
\end{abstract}

Keywords: Familial Hypertrophic Cardiomyopathy, Young Adult, Diagnosis, Low Income Country

\section{Introduction}

Hypertrophic cardiomyopathy (HCM) is a genetic disorder that is characterized by left ventricular hypertrophy unexplained by secondary causes such as hypertension or aortic stenosis with generally preserved or increased ejection fraction [1]. It is an autosomal dominant disease cause by mutation in cardiac muscle gene in around $60 \%$ of adult and adolescent. In 5 to $10 \%$ of adult, it is usually caused by other genetic disorders including metabolic and neuromuscular diseases, chromosomal abnormalities and genetics syndromes. Some patients have non-genetic disorders that mimic genetic forms of the disease, for example amyloidosis [2]. The genes responsible for familial HCM induce production of proteins that play important role in contraction of heart muscle. The proteins will combine to form sarcomeres, which are necessary for muscle contractions. A mutation in these genes cause abnormal function of sarcomeres or reduce the amount of proteins made. When the function of sarcomeres is impaired, there is a disruption in the normal heart contraction [3]. Usually, familial HCM is commonly asymmetrical with the most severe hypertrophy involving the basal interventricular septum, the apical 
segment is rarely involving [1].

The prevalence of HCM is estimated in diverse studies in North America, Europe, Asia and Africa to be between 1 in 500 peoples $(0,2 \%)$ and 1 in $3000(0,03 \%)$. It occurs equally in both sexes with similar clinical and genetic features [4]. HCM is associated with sudden cardiac death in around 51\% of young patients aged 25 to 65 years. Other causes of death are progressive heart failure $(36 \%)$ and atrial fibrillation with its related complications $(13 \%)$ [5].

Several mutations have been identified like those encoding for the beta-myosin heavy chain (MYH7) and myosinbinding protein $\mathrm{C}$ (MYBPC3) which account for the majority of cases. Less concerned genes are cardiac troponin I and $\mathrm{T}$ (TNN13, TNNT2), tropo myosin alpha-1 chain (TPM1) and myosin light chain 3 (MYL3). Patient with familial history of HCM due to sarcomere protein mutation have higher risk of sudden cardiac death than those without mutation [5]. This high risk population is rarely identified in low income countries because of unavailability of various tests. We present a special case of familial apical hypertrophic cardiomyopathy in a young male of Cameroonian origin identified by all required morphological and genetic exams.

\section{Case presentation}

A 36 years old male accountant, with unknown family history of sudden death and no cardiovascular risk factors presents in our cardiology unit with a chronic history of progressive chest pain on exertion, constrictive in nature, intermittent of about 5 minutes duration, non-radiating. There was no associated respiratory dyspnea, pedal edema or syncope.

Physical examination showed normal vital parameters. The first and second heart sounds were normal and regular; the apical precordial impulse was not displaced. The pulmonary exam was normal. Double or triple apical impulse was not found during cardiac examination.

Resting ECG showed sinus regular rhythm with a heart rate of 76 beats per minute, left ventricular hypertrophy with systolic overload, inverted $\mathrm{T}$ waves in lateral, inferior and anterior lead (Figure 1).

The cardiac ultrasound showed an apical hypertrophy with a dimension of $168 \mathrm{~mm}$, a non-dilated left ventricle and conserved left ventricular systolic function. The mitral profile and left ventricular filling pressure were normal (Figure 2). A diagnosis of probable ischemic disease was made based on the chest pain and inverted $\mathrm{T}$ waves. Cardiac biomarkers where negative, treadmill electrocardiography was normal also with coronarography

Considering all these findings, a diagnosis of an apical hypertrophic cardiomyopathy was made. Genetic test done in "Hôpital Europeen Georges-Pompidou" in France showed substitution of the 18 axon of gene MYBPC3 (c.1731G>A; p. Trp577*) leading to a premature stop code. Screening cardiac ultrasound was done for his siblings, parents, aunts and uncles of the maternal family since the patient didn't have any contact with his paternal family. The cardiac ultrasound was normal in all family members. However, his 16 years old son had a hypertrophy of the septum estimated at $14 \mathrm{~mm}$. The patient was treated with Propanolol 40mg: 1 tablet twice daily and paracetamol 500mg, 2 tablets three times a day, with improvement of patient symptoms. This treatment reduces the pain and ventricular contractility while increasing ventricular volume. An annual cardiac ultrasound controlled was scheduled for the patient and his family.

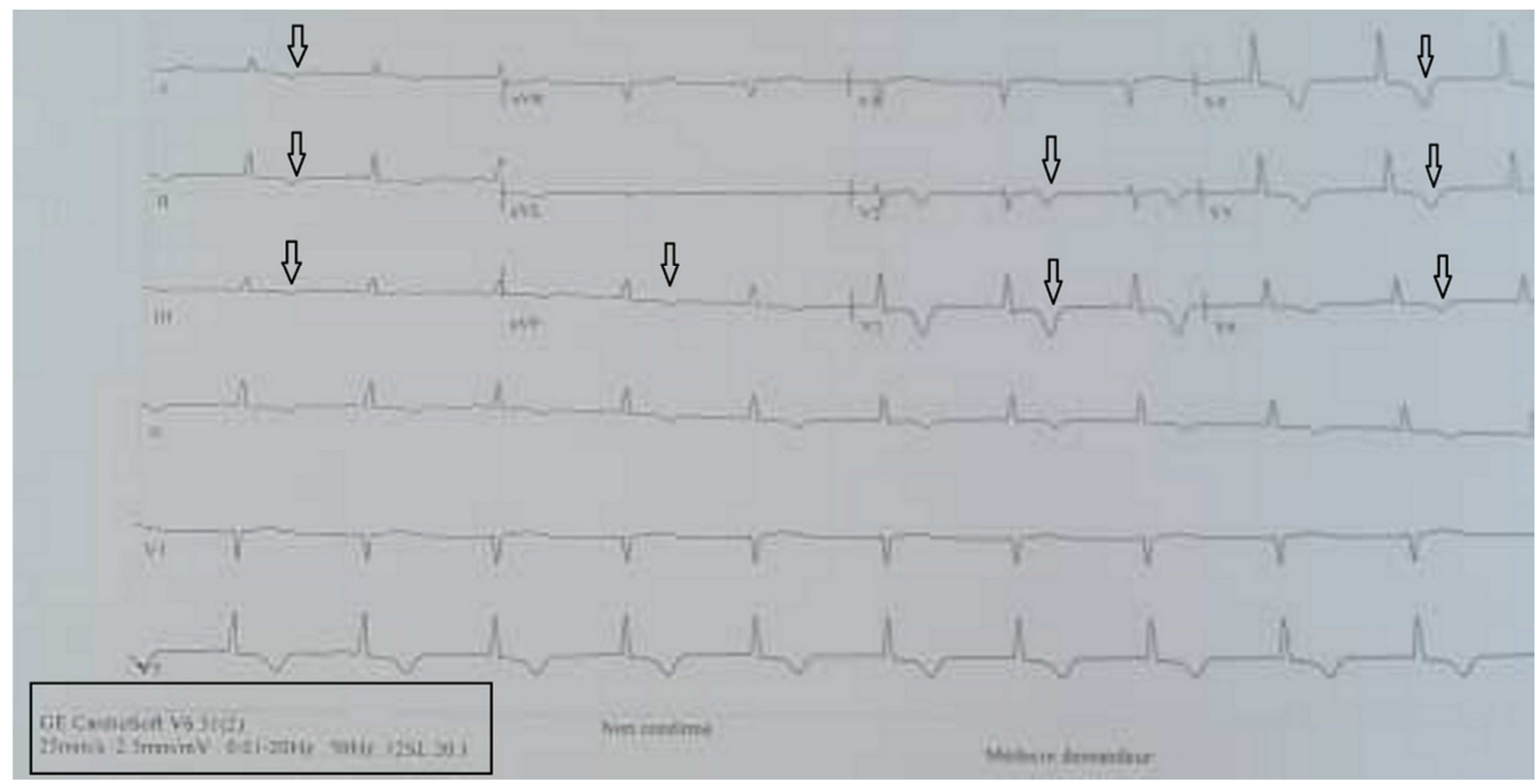

Figure 1. Left ventricular hypertrophy with systolic overload; inverted T waves in lateral, inferior and anterior leads. 


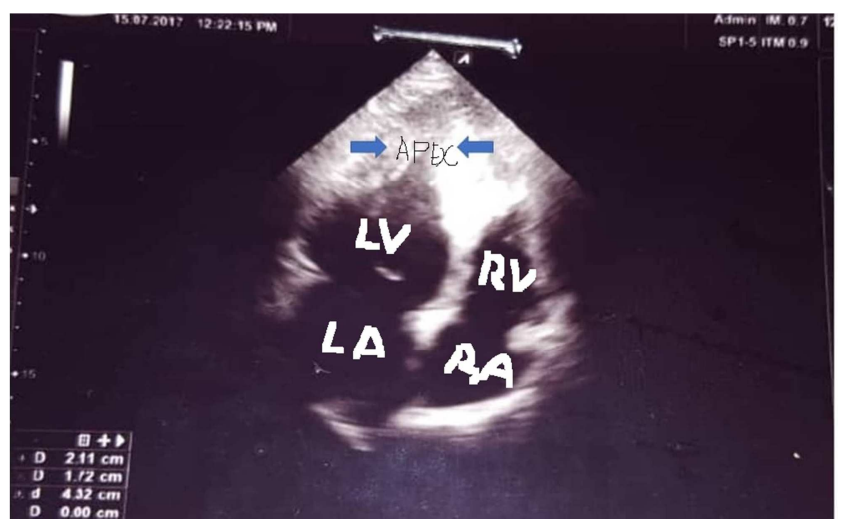

\$ LV: Left ventricle, LA: Left atrium; RV: Right Ventricle; RA: Right Atrium

Figure 2. Apical hypertrophic cardiomyopathy with non-dilated cardiac cavities.

\section{Discussion}

Apical HCM is a rare presentation of HCM [1]. This condition was first described in Japanese males in 1976 [6]. Usually it present with little or no symptoms [7]. The most common presentation is atypical chest pain [7]. It can also present with sudden cardiac death, dyspnea, and syncope [89]. The patient presented with resting chest pain similar to an acute coronary syndrome. But we ruled out this possibility with normal cardiac enzymes and coronarography. This pain is due to a disturbance between myocardial oxygen demand and blood supplies. The other symptoms were not present because of the topography of the hypertrophy. This electrocardiographic pattern is frequent in apical HCM, it is known as giant negative $\mathrm{T}$ wave. It is specific for apical HCM due to distal hypertrophy [10].

The physical examination in case of apical HCM is generally poor, with occasional discovering of a left ventricular heave or loud fourth heart sound contrary to classical HCM which has well-described characteristic physical findings [7-8].

The genetic test that was performed showed an anomaly in the MYBPC3 gene, this is the most frequent genetic abnormality recorded [3].

Apical HCM generally has a more benign course compared to other variants of HCM and is usually not associated with significant outflow tract obstruction. Just like classical HCM, they have a slight increased risk of arrhythmias [7].

During the follow up of the patient, we did several ECG and echocardiography; we didn't find evidence of myocardial ischemia or significant arrhythmia. His 6years follow-up electrocardiograms were relatively stable. Ventricular arrhythmia is one of the major natural evolution of the disease described by Nakamura et al. in Japan in 1990 [11] and which requires sometimes implantable cardiac devices.

This case offers the possibility to describe a rare presentation of familial hypertrophic cardiomyopathy using highly sophisticated exams in a low income country.

\section{Conclusion}

Familial Hypertrophic Cardiomyopathy is a rare disease; the precise diagnosis requires complex exams which are sometimes unavailable in low income countries. This case was interesting because apical presentation is rare and all necessary investigations were done for this patient giving the possibility to organize follow-up of patient and related family member to prevent sudden death.

\section{Abbreviations}

\author{
ECG: Electrocardiography \\ HCM: Hypertrophic cardiomyopathy \\ MYBP: myosin binding protein \\ MYH: myosin light chain \\ TPM: tropomyosin alpha- 1 chain
}

\section{Authors' Contributions}

Ndongo Sylvie Amougou managed the patient. Helles Murielle Lema and Ngam Mary Engonwei drafted the initial manuscript which was modified by Mazou Ngou Temgoua. Samuel Kingue supervised the Case. All authors read and approved the final manuscript.

\section{Consent for Publication}

Written informed consent was obtained from the patient for publication of this case report and any accompanying images. A copy of the written consent is available for review by the Editor-in-chief of this journal.

\section{Competing Interests}

The authors declare that they have no competing interests

\section{References}

[1] Marian A, Braunwald E. hypertrophic cardiomyopathy genetics, pathogenesis, clinical manifestations, diagnosis and therapy. Circres 2017; 121: 749-770.

[2] Elliott PM, Anastasakis A, Borger MA, et al. 2014 ESC Guidelines on diagnosis and management of hypertrophic cardiomyopathy: The Task force for the diagnosis and management of hypertrophic cardiomyopathy of the European Society of Cardiology (ESC). EurHeart J. 2014 Oct 14; 35 (39): 2733-79.

[3] Familial hypertrophic cardiomyopathy. Genetics Home Reference. August, 2015; http://ghr.nlm.nih.gov/condition/familial-hypertrophiccardiomyopathy.

[4] Barry J. Maron, Steve R. ommen et al, Hypertrophic cardiomyopathy present and future, with translation into contemporary cardiovascular medecine. Jacc. 2014. 64:0735-1097.

[5] Barry J. Maron, Iacopo Olivotto et al, Epidemiology of cardiomyopathy-relateddeathrevisited in a large nonreferralbased population. CIR. 2000; 102: 858-864. 
[6] Sakamoto $\mathrm{T}$, Tei $\mathrm{C}$ et al. Giant $\mathrm{T}$ wave inversion as a manifestation of asymmetrical apical hypertrophy of the left ventricle: echocardiographic and ultrasonocardiotomographicstudy.jpn Heart J. 1976; 17: 611629.

[7] Ting P, Gunasegaran K, Teo W S. Electrocardiographicalcase. asymptomatic patient with deep T-wave inversions. Singapore Med J 2007; 48 (6): 586

[8] Jeffrey B. Geske, Steve R. Ommen et al. Hypertrophic cardiomyopathy clinical update. JACC: heart failure. 2018 May 5.6 (5): 364-75.
[9] YusufKasirye, Janaki R Manne et al. Apical hypertrophic cardiomyopathy presenting as recurrent unexplained syncope. Clin Med Res. 2012 Feb; 10 (1): 26-31

[10] Carlos A. Dumont, Lorenzo Monserrat et al. Interpretation of electrocardiographic abnormalities in hypertrophic cardiomyopathy with cardiac magnetic resonance. Eur Heart J. 2006; 27: 1725-1731

[11] Nakamura T, Furukawa K et al. Long-term follow-up of electrocardiographic changes in patients with assymetric apical hypertrophy. J Cardiol. 1990; 20 (3): 635-47. 